United Nations Educational Scientific and Cultural Organization and

International Atomic Energy Agency

THE ABDUS SALAM INTERNATIONAL CENTRE FOR THEORETICAL PHYSICS

\title{
ON THE CONTINUOUS SELECTIONS OF SOLUTION SETS OF LIPSCHITZIAN QUANTUM STOCHASTIC DIFFERENTIAL INCLUSIONS
}

\author{
E.O. Ayoola ${ }^{1}$ \\ Department of Mathematics, University of Ibadan, Ibadan, Nigeria \\ and \\ The Abdus Salam International Centre for Theoretical Physics, Trieste, Italy.
}

\begin{abstract}
We prove that a multifunction associated with the set of solutions of Lipschitzian quantum stochastic differential inclusion (QSDI) admits a selection continuous from some subsets of complex numbers to the space of the matrix elements of adapted weakly absolutely continuous quantum stochastic processes. In particular, we show that the solution set map as well as the reachable set of the QSDI admit some continuous representations.
\end{abstract}

MIRAMARE - TRIESTE

May 2004

\footnotetext{
${ }^{1}$ Regular Associate of the Abdus Salam ICTP. eoayoola@ictp.trieste.it
} 


\section{INTRODUCTION}

This paper is concerned with the problems of continuous selections of multivalued solution maps of quantum stochastic differential inclusions in integral form, given by

$$
\begin{aligned}
X(t) \in & a+\int_{0}^{t} E(s, X(s)) d \wedge_{\pi}(s)+F(s, X(s)) d A_{f}(s) \\
& +G(s, X(s)) d A_{g}^{+}(s)+H(s, X(s)) d s, \quad \text { almost all } t \in[0, T] .
\end{aligned}
$$

QSDI (1.1) is understood in the framework of the Hudson and Parthasarathy (1984) formulation of Boson quantum stochastic calculus. In the notations and definitions of various spaces of stochastic processes introduced in the work of Ekhaguere (1992), the coefficients $E, F, G, H$, lie in $L_{l o c}^{2}([0, T] \times \tilde{\mathcal{A}})_{m v s}$, where $\tilde{\mathcal{A}}$ is a locally convex space and $(0, a) \in[0, T] \times \tilde{\mathcal{A}}$ is a fixed point. The maps $f ; g, \pi$ appearing in (1.1) lie in some suitable function spaces. The integrators $\wedge_{\pi}, A_{g}^{+}$and $A_{f}$ are the gauge, creation and annihilation processes associated with the basic field operators of quantum field theory. As in our previous works (Ayoola (2003a, 2003b, 2001)) concerning some approximation of the reachable sets and solutions of QSDI (1.1), we consider the equivalent form of (1.1) given by

$$
\begin{aligned}
\frac{d}{d t}<\eta, X(t) \xi> & \in P(t, X(t))(\eta, \xi) \\
X(0) & =a, \quad t \in[0, T] .
\end{aligned}
$$

Inclusion (1.2) is a nonclassical ordinary differential inclusion and the map $(\eta, \xi) \rightarrow P(t, x)(\eta, \xi)$ is a multivalued sesquilinear form on $(\mathbb{D} \underline{\otimes} \mathbb{E})^{2}$ for $(t, x) \in[0, T] \times \tilde{\mathcal{A}}$. We refer the reader to the works of Ekhaguere $(1992,1995,1996)$ for the explicit forms of the map and the existence results for solutions of QSDI (1.1) of Lipschitz, hypermaximal monotone and of evolution types. We follow the fundamental concepts and structures as in the references by employing the locally convex space $\tilde{\mathcal{A}}$ of noncommutative stochastic processes whose topology is generated by the family of seminorms $\left\{\|x\|_{\eta \xi}=\mid\langle\eta, x \xi>|, \quad x \in \mathcal{A}, \eta, \xi \in \mathbb{D} \underline{\otimes} \mathbb{E}\right\}$. Here, as usual, the underlying elements of $\tilde{\mathcal{A}}$ consist of linear maps from $\mathbb{D} \underline{\otimes} \mathbb{E}$ into $\mathcal{R} \otimes \Gamma\left(L_{\gamma}^{2}\left(\mathbb{R}_{+}\right)\right)$having domains of their adjoints containing $\mathbb{D} \underline{\otimes} \mathbb{E}$. In particular, the spaces $L_{l o c}^{P}(\tilde{\mathcal{A}}), \quad L_{\gamma, l o c}^{\infty}\left(\mathbb{R}_{+}\right), \quad L_{l o c}^{P}(I \times \tilde{\mathcal{A}})$ for a fixed Hilbert space $\gamma$ are being adopted as in the above references.

In what follows, we consider QSDI (1.2) where the map $x \rightarrow P(t, x)(\eta, \xi)$ is Lipschitzian with values that are closed (not necessarily convex nor bounded) subsets of the field of complex numbers. The point $a$ ranges in a subset $A$ of $\tilde{\mathcal{A}}$ such that the set $A(\eta, \xi):=\{\langle\eta, a \xi\rangle: a \in A\}$ is compact in $\mathbb{C}$.

We denote by $S^{(T)}(a)$ the map that assigns to each point $a \in A$, the set of solutions of QSDI (1.2) and prove a continuous selection theorem from the map $S^{(T)}(a)(\eta, \xi)$ where

$$
S^{(T)}(a)(\eta, \xi)=\left\{<\eta, \Phi(\cdot) \xi>/ \Phi \in S^{(T)}(a)\right\}
$$


An important consequence of our main result is that the set map $<\eta, a \xi>\rightarrow S^{(T)}(a)(\eta, \xi)$ can be continuously represented in the

$$
g(<\eta, a \xi>, \mathcal{U})=S^{(T)}(a)(\eta, \xi) .
$$

Similar result holds for the case of the map from $<\eta, a \xi>$ to the set $R^{(T)}(a)(\eta, \xi)$, where

$$
R^{(T)}(a)(\eta, \xi)=\left\{<\eta, \Phi(T) \xi>/ \Phi(T) \in R^{(T)}(a)\right\}
$$

is the set of complex numbers associated with the reachable set

$$
R^{(T)}(a)=\left\{\Phi(T) / \Phi \in S^{(T)}(a)\right\}
$$

of QSDI (1.1) at time T (see our previous work Ayoola (2003b) for details).

Our results in this work are extensions of the results of Cellina and Ornelas (1992) to the present noncommutative quantum setting involving inclusions in certain locally convex spaces. We adapt the arguments employed in the reference to conform with our noncommutative stochastic analysis.

Problems of continuous selections of classical differential inclusions have attracted considerable attention in the literature. Some selection results at the classical setting can be found in the works of Cellina (1988), Aubin and Cellina (1984), Fryszkowski (1983), Antosiewicz and Cellina (1975), Colombo et al (1991) and the book by Repovs and Semenov (1998). As shown in the work of Cellina and Ornelas (1992) selection results have been used to show that the solution set - map and the attainable set admit some continuous parameterizations. Broucke and Arapostathis (2001) have established the existence of a continuous selection from the set of solutions that interpolates a given finite set of trajectories of Lipschitz differential inclusion.

The rest of the paper is organised as follows: In Section 2, we outline some fundamental definitions, notations and results concerning the selection results. Section 3 is devoted to the establishment of the main results of the paper.

\section{NOTATIONS AND PRELIMINARY RESULTS}

We shall employ the following notations in what follows. If $\mathcal{N}$ is a topological space, then $\operatorname{clos}(\mathcal{N})(\operatorname{resp} \cdot \operatorname{comp}(\mathcal{N}))$ denotes the collection of all nonempty closed (resp. compact) subsets of $\mathcal{N}$. We shall employ the Hausdorff topology on $\operatorname{clos}(\tilde{\mathcal{A}})$ as explained in Ekhaguere (1992).

We denote by $\rho(A, B)$ the Hausdorff distance between the sets $A, B$ in $\operatorname{clos}(\mathbb{C})$. The distance $d(x, A)$ of a point x from a set $A \in \operatorname{clos}(\mathscr{C})$ is defined by

$$
d(x, A)=\inf \{|x-a|: a \in A\} .
$$

We denote by $I$, the interval $[0, T]$ and the characteristic function of a subset $E$ of $I$ by $\chi_{E}$. As explained in Ekhaguere (1992), we consider the space $\operatorname{wac}(\tilde{\mathcal{A}})$ the completion of the locally 
convex space $\left(\operatorname{Ad}(\tilde{\mathcal{A}})_{w a c}, \quad \tau^{w a c}\right)$ where the topology $\tau^{w a c}$ is generated by the family of seminorms $\left\{|\cdot|_{\eta \xi}: \eta, \xi \in \mathbb{D} \underline{\otimes} \mathbb{E}\right\}$ defined for each $\Phi \in \operatorname{Ad}(\tilde{\mathcal{A}})_{\text {wac }}$ by

$$
|\Phi|_{\eta \xi}=\|\Phi(0)\|_{\eta \xi}+\int_{0}^{T}\left|\frac{d}{d s}<\eta, \Phi(s) \xi>\right| d s .
$$

Associated with $\operatorname{wac}(\tilde{\mathcal{A}})$, we define for arbitrary $\eta, \xi \in \mathbb{D} \underline{\otimes} \mathbb{E}$, the space of complex valued functions

$$
\operatorname{wac}(\tilde{\mathcal{A}})(\eta, \xi)=\{<\eta, \Phi(\cdot) \xi>/ \Phi \in \operatorname{wac}(\tilde{\mathcal{A}})\} .
$$

We remark that each element $\Phi_{\eta \xi}(\cdot):=<\eta, \Phi(\cdot) \xi>$ of $\operatorname{wac}(\tilde{\mathcal{A}})(\eta, \xi)$ is an absolutely continuous complex valued function on the interval $[0, T]$. We assume that $A$ is a subset of $\tilde{\mathcal{A}}$ such that the set of complex numbers

$$
A(\eta, \xi)=\{<\eta, a \xi>/ a \in A\}
$$

is compact in $\mathbb{C}$ with diameter $D_{\eta \xi}=\sup _{x, y \in A(\eta, \xi)}|x-y|$.

Furthermore, the map $(t, x) \rightarrow P(t, x)(\eta, \xi)$ appearing in (1.2) is assumed to satisfy the following conditions.

$\mathcal{S}_{(i)}$ The values of $P(t, x)(\eta, \xi)$ are nonempty closed, subsets of the field $\mathbb{C}$ of complex numbers.

$\mathcal{S}_{(i i)}$ The map $t \rightarrow P(t, x)(\eta, \xi)$ is measurable.

$\mathcal{S}_{(i i i)}$ There exists a map $K_{\eta \xi}^{P}:[0, T] \rightarrow \mathbb{R}_{+}$lying in $L_{l o c}^{1}([0, T])$ such that

$$
\rho(P(t, x)(\eta, \xi), P(t, y)(\eta, \xi)) \leq K_{\eta \xi}^{P}(t)\|x-y\|_{\eta \xi}
$$

for $t \in[0, T]$, and for each pair $x, y \in \tilde{\mathcal{A}}$.

$\mathcal{S}_{(i v)}$ There exists a stochastic process $Y:[0, T] \rightarrow \tilde{\mathcal{A}}$ lying in $\operatorname{Ad}(\tilde{\mathcal{A}})_{\text {wac }}$ such that the map

$$
t \rightarrow d\left(\frac{d}{d t}<\eta, Y(t) \xi>, P(t, Y(t))(\eta, \xi)\right)
$$

lies in $L_{l o c}^{1}([0, T])$.

From the result of Ekhaguere (1992), it is known that under the conditions $\mathcal{S}_{(i)}$ to $\mathcal{S}_{(i v)}$, QSDI (1.1) admits at least one adapted weakly absolutely continuous solution for each $a \in A$. We denote the set of all such solutions, with the topology of $w a c(\tilde{\mathcal{A}})$ by $S^{(T)}(a)$.

To prove our main result in Section 3, we need an important notion of partition of unity. 
Definition 2.1 : Let $A$ be a subset of $\tilde{\mathcal{A}}$ such that for arbitrary elements $\eta, \xi \in \mathbb{D} \underline{\otimes} \mathbb{E}$, the set $A(\eta, \xi)$ is compact in the field of complex numbers.

Let $\left\{\Omega_{i}\right\}_{i \in J}$ be an open covering for $A(\eta, \xi)$ with a finite open subcovering $\left\{\Omega_{i}\right\}, i=1,2, \cdots m$. A family of functions $\left\{P_{i}(\cdot)\right\}, i=1,2 \cdots m$ defined on $A(\eta, \xi)$ is called a Lipschitzian partition of unity subordinated to the finite subcovering if:

(i) $P_{i}(\cdot)$ is Lipschitzian for all $i=1,2 \cdots m$. That is, there exists constant $L_{\eta \xi}$ such that for any pair $a_{\eta \xi}, a_{\eta \xi}^{\prime} \in A(\eta, \xi)$, we have

$$
\left|P_{i}\left(a_{\eta \xi}\right)-P_{i}\left(a_{\eta \xi}^{\prime}\right)\right| \leq L_{\eta \xi}\left|a_{\eta \xi}-a_{\eta \xi}^{\prime}\right| .
$$

(ii) $P_{i}\left(a_{\eta \xi}\right)>0$ for $a_{\eta \xi} \in \Omega_{i} \cap A(\eta, \xi)$ and $P_{i}\left(a_{\eta \xi}\right)=0$ for $a_{\eta \xi} \in A(\eta, \xi) \backslash \Omega_{i}$.

(iii) For each $a_{\eta \xi} \in A(\eta, \xi), \sum_{i=1}^{m} P_{i}\left(a_{\eta \xi}\right)=1$.

Lemma 2.1 Let $A \subseteq \tilde{\mathcal{A}}$ such that for arbitrary $\eta, \xi \in \mathbb{D} \underline{\otimes} \mathbb{E}$, the set $A(\eta, \xi)$ is compact in $\mathbb{C}$. Then there exists a Lipschitzian partition of unity subordinated to any finite subcovering of an open covering for the set $A(\eta, \xi)$.

Proof: Let $\left\{\Omega_{i}\right\}, i=1,2 \cdots m$ be a finite open subcovering of an open covering $\left\{\Omega_{i}\right\}_{i \in J}$ of $A(\eta, \xi)$ in the field of complex numbers. First, by Lemma 2.1 in Smirnov (2002) for $Q \subseteq \mathbb{C}$, the map $q: \mathbb{C} \rightarrow \mathbb{R}_{+}$defined by $q(x)=d(x, Q)$ satisfies for $x_{1}, x_{2} \in \mathbb{C}$

$$
\left|q\left(x_{1}\right)-q\left(x_{2}\right)\right| \leq\left|x_{1}-x_{2}\right|
$$

For $i=1,2 \cdots m$, define the functions $q_{i}: A(\eta, \xi) \rightarrow \mathbb{R}_{+}$by

$$
q_{i}\left(a_{\eta \xi}\right)=d\left(a_{\eta \xi}, A(\eta, \xi) \backslash \Omega_{i}\right)
$$

and functions $P_{i}: A(\eta, \xi) \rightarrow \mathbb{R}_{+}$by

$$
P_{i}\left(a_{\eta \xi}\right)=\frac{q_{i}\left(a_{\eta \xi}\right)}{\sum_{j=1}^{m} q_{j}\left(a_{\eta \xi}\right)}
$$

For at least one $j \in\{1,2 \cdots m\}, a_{\eta \xi} \in \Omega_{j}$. Therefore $\sum_{j=1}^{m} q_{j}\left(a_{\eta \xi}\right)>0$.

Consequently, (2.1) is well defined.

Moreover, for each $a_{\eta \xi} \in A(\eta, \xi), \sum_{i=1}^{m} P_{i}\left(a_{\eta \xi}\right)=1$ and $P_{i}\left(a_{\eta \xi}\right)>0$ for

$a_{\eta \xi} \in \Omega_{i} \cap(A(\eta, \xi))$, and $P_{i}\left(a_{\eta \xi}\right)=0$ for $a_{\eta \xi} \in A(\eta, \xi) \backslash \Omega_{i}$.

Next we show that each function $P_{i}$ is Lipschitzian on $A(\eta, \xi)$.

Since the set $A(\eta, \xi)$ is compact, there exist numbers $M_{\eta \xi}, m_{\eta \xi}>0$ such that

$$
m_{\eta \xi}<\sum_{j=1}^{m} q_{j}\left(a_{\eta \xi}\right)<M_{\eta \xi}
$$


for any element $a_{\eta \xi} \in A(\eta, \xi)$.

For any pair $a_{\eta \xi}, a_{\eta \xi}^{\prime} \in A(\eta, \xi)$, we have

$$
\begin{aligned}
& \left|P_{i}\left(a_{\eta \xi}\right)-P_{i}\left(a_{\eta \xi}^{\prime}\right)\right| \\
= & \frac{\left|q_{i}\left(a_{\eta \xi}^{\prime}\right) \sum_{j=1}^{m} q_{j}\left(a_{\eta \xi}\right)-q_{i}\left(a_{\eta \xi}\right) \sum_{j=1}^{m} q_{j}\left(a_{\eta \xi}^{\prime}\right)\right|}{\sum_{j=1}^{m} q_{j}\left(a_{\eta \xi}\right) \sum_{j=1}^{m} q_{j}\left(a_{\eta \xi}^{\prime}\right)} \\
\leq & \frac{\left|q_{i}\left(a_{\eta \xi}^{\prime}\right) \sum_{j=1}^{m} q_{j}\left(a_{\eta \xi}\right)-q_{i}\left(a_{\eta \xi}\right) \sum_{j=1}^{m} q_{j}\left(a_{\eta \xi}^{\prime}\right)\right|}{m_{\eta \xi}^{2}} \\
\leq & \frac{1}{m_{\eta \xi}^{2}} \sum_{j=1}^{m}\left(\left|q_{i}\left(a_{\eta \xi}^{\prime}\right) q_{j}\left(a_{\eta \xi}\right)-q_{i}\left(a_{\eta \xi}\right) q_{j}\left(a_{\eta \xi}\right)\right|\right. \\
+ & \left.\left|q_{i}\left(a_{\eta \xi}\right) q_{j}\left(a_{\eta \xi}\right)-q_{i}\left(a_{\eta \xi}\right) q_{j}\left(a_{\eta \xi}^{\prime}\right)\right|\right) \\
\leq & \frac{1}{m_{\eta \xi}^{2}}\left(\sum_{j=1}^{m} q_{j}\left(a_{\eta \xi}\right)\left|a_{\eta \xi}-a_{\eta \xi}^{\prime}\right|+q_{i}\left(a_{\eta \xi}\right) \sum_{j=1}^{m}\left|a_{\eta \xi}-a_{\eta \xi}^{\prime}\right|\right) \\
\leq & \frac{(1+m) M_{\eta \xi}}{m_{\eta \xi}^{2}}\left|a_{\eta \xi}-a_{\eta \xi}^{\prime}\right|,
\end{aligned}
$$

where we have used the inequality

$$
\left|q_{i}\left(a_{\eta \xi}^{\prime}\right)-q_{i}\left(a_{\eta \xi}\right)\right| \leq\left|a_{\eta \xi}^{\prime}-a_{\eta \xi}\right|
$$

satisfied by each function $q_{i}(\cdot), \quad i=1,2 \cdots m$ on $A(\eta, \xi)$.

Thus $P_{i}(\cdot)$ is Lipschitzian with Lipschitz constant $L_{\eta \xi}=\frac{(1+m) M_{\eta \xi}}{m_{\eta \xi}^{2}}$.

Next, we present a proposition which we shall frequently use in the proof of our selection theorem. We obtained the result by adapting its classical analogue presented in Cellina and Ornelas (1992) to the present noncommutative quantum setting.

Proposition 2.2. Let $V_{0}, V_{1}, \cdots V_{m}$ be stochastic processes in $L_{l o c}^{1}(\tilde{\mathcal{A}})$ and for any pair of points $\eta, \xi \in \mathbb{D} \underline{\otimes} \mathbb{E}$, let $\left\{I_{j}\left(a_{\eta \xi}\right)\right\}$ be a partition of the interval $I=[0, T]$ into a finite number of subintervals with endpoints depending continuously on the point $a_{\eta \xi}:=<\eta, a \xi>, a \in A$.

Consider the map

$$
W: a_{\eta \xi} \rightarrow a_{\eta \xi}+\int_{0}^{t} \sum_{j=0}^{m} \chi_{I_{j}\left(a_{\eta \xi}\right)}(s)<\eta, V_{j}(s) \xi>d s .
$$

Then there exists a map $R_{\eta \xi}(t)$ lying in $L_{l o c}^{1}([0, T])$ such that for every $\epsilon>0$, there exists $\partial>0$ such that $\left|a_{\eta \xi}-a_{\eta \xi}^{\prime}\right|<\partial$ implies that

$$
\left|\frac{d}{d t} W\left(a_{\eta \xi}\right)(t)-\frac{d}{d t} W\left(a_{\eta \xi}^{\prime}\right)(t)\right| \leq R_{\eta \xi}(t) \chi_{E}(t),
$$

for some set $E \subseteq I$ with measure $\mu(E)<\epsilon$. 
Proof: First, we assume the hypothesis of Lemma 2.1. By the conclusion of the lemma, there exists a Lipschitzian partition of unity $P_{j}(\cdot)$ subordinated to a finite open subcovering of $A(\eta, \xi)$.

Let $\epsilon>0$ be given. Define for each $a_{\eta \xi}$ in $A(\eta, \xi)$,

$$
t_{0}\left(a_{\eta \xi}\right)=0, \quad t_{j}\left(a_{\eta \xi}\right)=t_{j-1}\left(a_{\eta \xi}\right)+T P_{j}\left(a_{\eta \xi}\right), \quad 1 \leq j \leq m .
$$

Then for each $j, t_{j}$ is continuous on $A(\eta, \xi)$. This can be shown as follows:

For any pair $a_{\eta \xi}, \quad a_{\eta \xi}^{\prime} \in A(\eta, \xi)$ we have

$\left|t_{j}\left(a_{\eta \xi}\right)-t_{j}\left(a_{\eta \xi}^{\prime}\right)\right|$

$\leq\left|t_{j-1}\left(a_{\eta \xi}\right)-t_{j-1}\left(a_{\eta \xi}^{\prime}\right)\right|+T\left|P_{j}\left(a_{\eta \xi}\right)-P_{j}\left(a_{\eta \xi}^{\prime}\right)\right|$

$\leq\left|t_{j-1}\left(a_{\eta \xi}\right)-t_{j-1}\left(a_{\eta \xi}^{\prime}\right)\right|+T L_{\eta \xi}\left|a_{\eta \xi}-a_{\eta \xi}^{\prime}\right|$,

since each $P_{j}(\cdot)$ is Lipschitzian.

At the $j$ th iteration, we have

$$
\left|t_{j}\left(a_{\eta \xi}\right)-t_{j}\left(a_{\eta \xi}^{\prime}\right)\right| \leq j T L_{\eta \xi}\left|a_{\eta \xi}-a_{\eta \xi}^{\prime}\right|
$$

Putting $\delta_{j}=\frac{\epsilon}{j T L_{\eta \xi}}$, then whenever $\left|a_{\eta \xi}-a_{\eta \xi}^{\prime}\right| \leq \delta_{j}$, we have

$$
\left|t_{j}\left(a_{\eta \xi}\right)-t_{j}\left(a_{\eta \xi}^{\prime}\right)\right| \leq \epsilon .
$$

Now we define the intervals $I_{j}\left(a_{\eta \xi}\right)=\left[t_{j-1}\left(a_{\eta \xi}\right), t_{j}\left(a_{\eta \xi}\right)\right)$ for $j=0,1, \cdots m$. Thus

$$
I=[0, T]=\bigcup_{j=0}^{m} I_{j}\left(a_{\eta \xi}\right) .
$$

We observe that for any two points $a_{\eta \xi}, \quad a_{\eta \xi}^{\prime} \in A(\eta, \xi)$, we have the following estimate

$$
\begin{aligned}
& \left|\frac{d}{d t} W\left(a_{\eta \xi}\right)(t)-\frac{d}{d t} W\left(a_{\eta \xi}^{\prime}\right)(t)\right| \\
\leq & \sum_{j=0}^{m} \chi_{I_{j}\left(a_{\eta \xi}\right) \triangle I_{j}\left(a_{\eta \xi}^{\prime}\right)(t)}(t)\left\|V_{j}(t)\right\|_{\eta \xi} \\
= & \sum_{j=0}^{m} \chi_{I_{j}\left(a_{\eta \xi}\right) \triangle I_{j}\left(a_{\eta \xi}^{\prime}\right)}(t) R_{\eta \xi}(t)
\end{aligned}
$$

where $R_{\eta \xi}(t)=\max _{j}\left\|V_{j}(t)\right\|_{\eta \xi}$, for each $t \in[0, T]$ and

$$
\begin{aligned}
& I_{j}\left(a_{\eta \xi}\right) \triangle I_{j}\left(a_{\eta \xi}^{\prime}\right) \\
& =\left[I_{j}\left(a_{\eta \xi}\right) \cap\left(I_{j}\left(a_{\eta \xi}\right) \backslash I_{j}\left(a_{\eta \xi}^{\prime}\right)\right)\right] \bigcup\left[\left(I_{j}\left(a_{\eta \xi}^{\prime}\right) \backslash I_{j}\left(a_{\eta \xi}\right)\right) \cap I_{j}\left(a_{\eta \xi}^{\prime}\right)\right] .
\end{aligned}
$$


We remark that the family of sets $\left\{I_{j}\left(a_{\eta \xi}\right) \triangle I_{j}\left(a_{\eta \xi}^{\prime}\right)\right\}$ are pairwise disjoint. Putting $E=\bigcup_{j=0}^{m} I_{j}\left(a_{\eta \xi}\right) \triangle I_{j}\left(a_{\eta \xi}^{\prime}\right)$, then from (2.2),

$$
\left|\frac{d}{d t} W\left(a_{\eta \xi}\right)(t)-\frac{d}{d t} W\left(a_{\eta \xi}^{\prime}\right)(t)\right| \leq \chi_{E}(t) R_{\eta \xi}(t)
$$

where by the properties of characteristic functions (see for example, Halmos (1988)),

$$
\chi_{E}(t)=\sum_{j=1}^{m} \chi_{I_{j}\left(a_{\eta \xi}\right) \triangle I_{j}\left(a_{\eta \xi}^{\prime}\right)}(t) .
$$

Since the set $A(\eta, \xi)$ is compact in $\mathscr{C}$, then the family of functions $\left\{t_{j}\right\}$ is a uniformly equicontinuous family of real valued functions. Thus for every $\epsilon>0$, there exists $\delta>0$ such that for every $a_{\eta \xi}, a_{\eta \xi}^{\prime} \in A(\eta, \xi)$ satisfying

$$
\left|a_{\eta \xi}-a_{\eta \xi}^{\prime}\right|<\delta
$$

then

$$
\left|t_{j}\left(a_{\eta \xi}\right)-t_{j}\left(a_{\eta \xi}^{\prime}\right)\right|<\frac{\epsilon}{2(m+1)}
$$

Hence,

$$
\mu\left(I_{j}\left(a_{\eta \xi}\right) \triangle I_{j}\left(a_{\eta \xi}^{\prime}\right)\right) \leq 2\left|t_{j}\left(a_{\eta \xi}\right)-t_{j}\left(a_{\eta \xi}^{\prime}\right)\right| \leq \frac{\epsilon}{m+1}
$$

Consequently,

$$
\mu(E)=\sum_{j=0}^{m} \mu\left(I_{j}\left(a_{\eta \xi}\right) \triangle I_{j}\left(a_{\eta \xi}^{\prime}\right)\right)<\epsilon .
$$

\section{MAIN RESULTS}

We present in this section, our main results. In what follows, for points $a_{k} \in A, k=0,1,2 \cdots$, and $\Phi_{k} \in \mathrm{S}^{(T)}\left(a_{k}\right)$, we set $a_{\eta \xi, k}=<\eta, a_{k} \xi>$ and $\Phi_{\eta \xi, k}(\cdot)=<\eta, \Phi_{k}(\cdot) \xi>$.

Our method of proof for the main results below, is an adaptation of the arguments employed in Cellina and Ornelas (1992) concerning similar results for classical differential inclusions. In addition, we employ successive approximations similar to what we have in Ekhaguere (1992) for proving the existence of solutions of quantum stochastic differential inclusion (1.1).

\section{Theorem 3.1}

Assume that the map $(t, x) \rightarrow P(t, x)(\eta, \xi)$ satisfies conditions $\mathcal{S}_{(i)}-\mathcal{S}_{(i v)}$.

Let $\Phi_{0} \in \mathrm{S}^{(T)}\left(a_{0}\right)$ for a fixed point $a_{0} \in A$. Then there exists a continuous map $W: A(\eta, \xi) \rightarrow$ $\operatorname{wac}(\tilde{\mathcal{A}})(\eta, \xi)$, a selection from $\mathrm{S}^{(T)}(a)(\eta, \xi)$ such that

$$
W\left(a_{\eta \xi, 0}\right)=\Phi_{\eta \xi, 0}
$$

Proof: Since $\Phi_{0} \in \mathrm{S}^{(T)}\left(a_{0}\right)$, then $\Phi_{0} \in A d(\tilde{\mathcal{A}})_{w a c}$. By the properties of the solution established 
in Ekhaguere $(1992)$, there exists a stochastic process $V_{0}:[0, T] \rightarrow \tilde{\mathcal{A}}$ lying in $L_{\text {loc }}^{1}(\tilde{\mathcal{A}})$ such that for almost all $t \in[0, T]$,

$$
\Phi_{0}(t)=a_{0}+\int_{0}^{t} V_{0}(s) d s
$$

and for arbitrary $\eta, \xi \in \mathbb{D} \underline{\otimes} \mathbb{E}$, we have

$$
<\eta, V_{0}(s) \xi>=\frac{d}{d s}<\eta, \Phi_{0}(s) \xi>\in P\left(s, \Phi_{0}(s)\right)(\eta, \xi), \quad s \in[0, T] .
$$

Now for arbitrary element $a \in A$, set

$$
Y: A \rightarrow \operatorname{wac}(\tilde{\mathcal{A}})
$$

to be

$$
Y(a)(t)=a+\int_{0}^{t} V_{0}(s) d s .
$$

Associated with the map $Y$, we set the map

$$
W: A(\eta, \xi) \rightarrow \operatorname{wac}(\tilde{\mathcal{A}})(\eta, \xi)
$$

to be

$$
W\left(a_{\eta \xi}\right)(t)=a_{\eta \xi}+\int_{0}^{t}<\eta, V_{0}(s) \xi>d s
$$

We remark that the map $W$ is well defined and continuous on $A(\eta, \xi)$ and that

$$
\frac{d}{d t} W\left(a_{\eta \xi}\right)(t)=<\eta, V_{0}(t) \xi>
$$

Furthermore,

$$
\begin{aligned}
& d\left(\frac{d}{d t} W\left(a_{\eta \xi}\right)(t), P(t, Y(a)(t))(\eta, \xi)\right) \\
= & d\left(\frac{d}{d t}<\eta, \Phi_{0}(t) \xi>, P(t, Y(a)(t))(\eta, \xi)\right) \\
\leq & \rho\left(P\left(t, Y\left(a_{0}\right)(t)\right)(\eta, \xi), P(t, Y(a)(t))(\eta, \xi)\right) \\
\leq & K_{\eta \xi}^{P}(t)\left\|Y\left(a_{0}\right)(t)-Y(a)(t)\right\|_{\eta \xi} \\
= & K_{\eta \xi}^{P}(t)\left\|a_{0}-a\right\|_{\eta \xi} .
\end{aligned}
$$

Since the map $t \rightarrow P(t, Y(a)(t))(\eta, \xi)$ is measurable with closed values in the complex field, then by Theorem 2, Chapter 1, section 14 in the book of Aubin and Cellina (1984) (see also Ekhaguere $(1992))$, we can choose $U_{0}(a)(t)(\eta, \xi)$ to be a measurable selection from $P(t, Y(a)(t))(\eta, \xi)$ such that

$$
\begin{aligned}
& \left|\frac{d}{d t} W\left(a_{\eta \xi}\right)(t)-U_{0}(a)(t)(\eta, \xi)\right| \\
= & d\left(\frac{d}{d t} W\left(a_{\eta \xi}\right)(t), P(t, Y(a)(t))(\eta, \xi)\right) \\
\leq & K_{\eta \xi}^{P}(t)\left\|a_{0}-a\right\|_{\eta \xi} .
\end{aligned}
$$


As the map $(\eta, \xi) \rightarrow U_{0}(a)(t)(\eta, \xi)$ is a sesquilinear form on $\mathbb{D} \underline{\otimes} \mathbb{E}$ for almost all $t \in[0, T]$ and by the adaptedness of $Y(a)$, there exists an adapted stochastic process $U_{0}(a):[0, T] \rightarrow \tilde{\mathcal{A}}$ such that

$$
<\eta, U_{0}(a)(t) \xi>=U_{0}(a)(t)(\eta, \xi) .
$$

By equation (3.2), the process $U_{0}(a)$ lies in $L_{l o c}^{1}(\tilde{\mathcal{A}})$, for any $a \in A$. This assertion follows from the fact that from $(3.2)$, we have

$$
\left\|V_{0}(t)-U_{0}(a)(t)\right\|_{\eta \xi} \leq K_{\eta \xi}^{P}(t)\left\|a_{0}-a\right\|_{\eta \xi}
$$

where $V_{0} \in L_{l o c}^{1}(\tilde{\mathcal{A}})$.

Next we fix some positve real number $\theta$ and define for any

$a_{\eta \xi} \in A(\eta, \xi)$,

$$
\delta\left(a_{\eta \xi}\right)=\min \left\{2^{-3} \theta, \frac{\left|a_{\eta \xi}-a_{\eta \xi, 0}\right|}{2}\right\} a_{\eta \xi} \neq a_{\eta \xi, 0}
$$

and

$$
\delta\left(a_{\eta \xi, 0}\right)=2^{-3} \theta
$$

Next, we define the open balls

$$
B\left(a_{\eta \xi}, \quad \delta\left(a_{\eta \xi}\right)\right)=\left\{x \in \mathbb{C} /\left|x-a_{\eta \xi}\right|<\delta\right\} .
$$

Then the family of open sets $\left\{B\left(a_{\eta \xi}, \delta\left(a_{\eta \xi}\right)\right), a_{\eta \xi} \in A(\eta, \xi)\right\}$ covers the set $A(\eta, \xi)$.

By the compactness of $A(\eta, \xi)$ let $B\left(a_{\eta \xi, j}, \delta\left(a_{\eta \xi, j}\right)\right), \quad j=0,1,2 \cdots m$ be a finite open subcovering. We notice that the point $a_{\eta \xi, 0}$ belongs only to the set $B\left(a_{\eta \xi, 0}, \delta\left(a_{\eta \xi, 0}\right)\right)$.

Let $P_{j}(\cdot), j=0,1,2 \cdots m$ be a Lipschitzian partition of unity subordinate to the covering. We define the following intervals:

$$
I_{0}\left(a_{\eta \xi}\right)=\left[0, T P_{0}\left(a_{\eta \xi}\right)\right]
$$

and for $j>0$,

$$
I_{j}\left(a_{\eta \xi}\right)=\left[T\left(P_{0}\left(a_{\eta \xi}\right)+\cdots+P_{j-1}\left(a_{\eta \xi}\right)\right), T\left(P_{0}\left(a_{\eta \xi}\right)+\cdots P_{j}\left(a_{\eta \xi}\right)\right)\right]
$$

Next we set

$$
Y_{1}(a)(t)=a+\int_{0}^{t} \sum_{j=0}^{m} \chi_{I_{j}\left(a_{\eta \xi}\right)}(s) U_{0}\left(a_{j}\right)(s) d s
$$

and

$$
W_{1}\left(a_{\eta \xi}\right)(t)=a_{\eta \xi}+\int_{0}^{t} \sum_{j=0}^{m} \chi_{I_{j}\left(a_{\eta \xi}\right)}(s)<\eta, U_{0}\left(a_{j}\right)(s) \xi>d s .
$$

By Proposition (2.1), the map $W_{1}: A(\eta, \xi) \rightarrow \mathrm{w} a c(\tilde{\mathcal{A}})(\eta, \xi)$ is continuous. Moreover, since $a_{\eta \xi, 0}$ belongs only to the set $B\left(a_{\eta \xi, 0}, \delta\left(a_{\eta \xi, 0}\right)\right)$, 
$P_{0}\left(a_{\eta \xi, 0}\right)=1$ and therefore we have $I_{0}\left(a_{\eta \xi, 0}\right)=\left[\begin{array}{ll}0, & T\end{array}\right]$.

Since $\chi_{I_{j}\left(a_{\eta \xi, 0}\right)} U_{0}\left(a_{j}\right)(s)=0, \quad j \neq 0$, we have from (3.3) and (3.4),

$$
Y_{1}\left(a_{0}\right)(t)=a_{0}+\int_{0}^{t} U_{0}\left(a_{0}\right)(s) d s
$$

and

$$
W_{1}\left(a_{\eta \xi, 0}\right)(t)=a_{\eta \xi, 0}+\int_{0}^{t}<\eta, U_{0}\left(a_{0}\right)(s) \xi>d s .
$$

But by (3.2),

$$
\left|\frac{d}{d t} W\left(a_{\eta \xi, 0}\right)(t)-<\eta, U_{0}\left(a_{0}\right)(t) \xi>\right|=0
$$

That is

$$
\left|<\eta,\left(V_{0}(t)-U_{0}\left(a_{0}\right)(t)\right) \xi>\right|=0
$$

holds for arbitrary $\eta, \xi \in \mathbb{D} \underline{\otimes} \mathbb{E}$.

Hence,

$$
V_{0}(t)=U_{0}\left(a_{0}\right)(t), \quad t \in[0, T] .
$$

Therefore by (3.1)

$$
Y_{1}\left(a_{0}\right)(t)=\Phi_{0}(t)
$$

and

$$
W_{1}\left(a_{\eta \xi, 0}\right)(t)=\Phi_{\eta \xi, 0}(t)
$$

Next we have by (3.2)

$$
\begin{aligned}
& \int_{0}^{t}\left|\frac{d}{d s} W_{1}\left(a_{\eta \xi}\right)(s)-\frac{d}{d s} W\left(a_{\eta \xi}\right)(s)\right| d s \\
& \leq \int_{0}^{t} \sum_{j=0}^{m} \chi_{I_{j}\left(a_{\eta \xi}\right)}(s)\left|<\eta, U_{0}\left(a_{j}\right)(s) \xi>-\frac{d}{d s} W\left(a_{\eta \xi}\right)(s)\right| d s \\
& \leq \int_{0}^{t} \sum_{j=0}^{m} \chi_{I_{j}\left(a_{\eta \xi}\right)} K_{\eta \xi}^{P}(s)\left\|a_{0}-a_{j}\right\|_{\eta \xi} d s \leq D_{\eta \xi} M_{\eta \xi}(t)
\end{aligned}
$$

where

$$
M_{\eta \xi}(t)=\int_{0}^{t} K_{\eta \xi}^{P}(s) d s
$$

and

$$
\sum_{j=0}^{m} \chi_{I_{j}\left(a_{\eta \xi}\right)}(s)=\chi_{I}(s)=1, \quad I=\bigcup_{j=0}^{m} I_{j}\left(a_{\eta \xi}\right)=[0, T] .
$$

Let $t \in[0, T]$ be fixed and let $j \in\{0,1,2 \cdots m\}$ be such that $t \in I_{j}\left(a_{\eta \xi}\right)$. Then by the definition of $W_{1}\left(a_{\eta \xi}\right)(t)$,

$$
\begin{aligned}
& d\left(\frac{d}{d t} W_{1}\left(a_{\eta \xi}\right)(t), P(t, Y(a)(t))(\eta \xi)\right) \\
= & d\left(<\eta, U_{0}\left(a_{j}\right)(t) \xi>, P(t, Y(a)(t))(\eta, \xi)\right) \\
\leq & \rho\left(P\left(t, Y\left(a_{j}\right)(t)\right)(\eta, \xi), P(t, Y(a)(t))(\eta, \xi)\right)
\end{aligned}
$$




$$
\begin{aligned}
& \leq K_{\eta \xi}^{P}(t)\left\|Y\left(a_{j}\right)(t)-Y(a)(t)\right\|_{\eta \xi} \\
& =K_{\eta \xi}^{P}(t)\left\|a_{j}-a\right\|_{\eta \xi}=K_{\eta \xi}^{P}(t)\left|a_{\eta \xi, j}-a_{\eta \xi}\right| \\
& \leq 2^{-3} \theta K_{\eta \xi}^{P}
\end{aligned}
$$

We remark here that inequality (3.7) holds since $a_{\eta \xi} \in B\left(a_{\eta \xi, j}, \delta\left(a_{\eta \xi, j}\right)\right)$ for some $0 \leq j \leq m$ and $\left|a_{\eta \xi}-a_{\eta \xi, j}\right|<\delta\left(a_{\eta \xi, j}\right) \leq 2^{-3} \theta$.

The estimate holds on the whole interval $[0, T]$ since it is independent of $j$.

Similarly, we have for $t \in I_{j}\left(a_{\eta \xi}\right)$,

$$
\begin{aligned}
& d\left(\frac{d}{d t} W_{1}\left(a_{\eta \xi}\right)(t), P\left(t, Y_{1}(a)(t)\right)(\eta, \xi)\right) \\
\leq & d\left(\frac{d}{d t} W_{1}\left(a_{\eta \xi}\right)(t), P(t, Y(a)(t))(\eta, \xi)\right) \\
& +\rho\left(P(t, Y(a)(t))(\eta, \xi), P\left(t, Y_{1}(a)(t)\right)(\eta, \xi)\right) \\
\leq & K_{\eta \xi}^{P}(t) 2^{-3} \theta+K_{\eta \xi}^{P}(t)\left\|Y(a)(t)-Y_{1}(a)(t)\right\|_{\eta \xi} \\
\leq & K_{\eta \xi}^{P}(t)\left[2^{-3} \theta+D_{\eta \xi} M_{\eta \xi}(t)\right]
\end{aligned}
$$

Inequality (3.8) follows from the following estimates:

$$
\begin{aligned}
& \left\|Y(a)(t)-Y_{1}(a)(t)\right\|_{\eta \xi}=\left|W\left(a_{\eta \xi}\right)(t)-W_{1}\left(a_{\eta \xi}\right)(t)\right| \\
\leq & \int_{0}^{t}\left|\frac{d}{d s} W\left(a_{\eta \xi}\right)(s)-<\eta, U_{0}\left(a_{j}\right)(s) \xi>\right| d s \\
\leq & \int_{0}^{t} K_{\eta \xi}^{P}(s)\left\|a_{0}-a_{j}\right\|_{\eta \xi} \leq D_{\eta \xi} M_{\eta \xi}(t)
\end{aligned}
$$

by $(3.2)$

In general, we claim that for $n=1,2 \cdots$, we can define sequences of maps: $Y_{n}: A \rightarrow$ wac $(\tilde{\mathcal{A}})$ and $W_{n}: A(\eta, \xi) \rightarrow \operatorname{wac}(\tilde{\mathcal{A}})(\eta, \xi)$ such that $W_{n}$ is continuous on $A(\eta, \xi)$ and for each $a \in A$, $a_{\eta \xi} \in A(\eta, \xi), Y_{n}(a)$ lies in $L_{l o c}^{1}(\tilde{\mathcal{A}}), Y_{n}\left(a_{0}\right)=\Phi_{0}$ and $W_{n}\left(a_{\eta \xi, 0}\right)=\Phi_{\eta \xi, 0}$.

Moreover,

(i) $\quad \int_{0}^{t}\left|\frac{d}{d s} W_{n}\left(a_{\eta \xi}\right)(s)-\frac{d}{d s} W_{n-1}\left(a_{\eta \xi}\right)(s)\right| d s$

$$
\leq D_{\eta \xi} \frac{M_{\eta \xi}^{n}(t)}{n !}+\theta 2^{-n-1}\left[2^{-2}+\sum_{i=1}^{n} \frac{\left(2 M_{\eta \xi}(t)\right)^{i}}{i !}\right]
$$

(ii) $\quad d\left(\frac{d}{d t} W_{n}\left(a_{\eta \xi}\right)(t), P\left(t, Y_{n-1}(a)(t)\right)(\eta, \xi)\right) \leq \theta 2^{-n-2} K_{\eta \xi}^{P}(t)$

(iii) $\quad d\left(\frac{d}{d t} W_{n}\left(a_{\eta \xi}\right)(t), P\left(t, Y_{n}(a)(t)\right)(\eta, \xi)\right)$

$$
\leq D_{\eta \xi} K_{\eta \xi}^{P}(t) \frac{M_{\eta \xi}^{n}(t)}{n !}+\theta 2^{-n-1} K_{\eta \xi}^{P}(t) \sum_{i=0}^{n} \frac{\left(2 M_{\eta \xi}(t)\right)^{i}}{i !} .
$$


(iv) There exists maps $R_{\eta \xi, n}:[0, T] \rightarrow \mathbb{R}_{+}$lying in $L_{l o c}^{1}([0, T])$ such that for every $\epsilon>0$, there exists $\delta>0$, such that $\left|a_{\eta \xi}^{\prime}-a_{\eta \xi}\right|<\delta$ implies that

$$
\left|\frac{d}{d t} W_{n}\left(a_{\eta \xi}\right)(t)-\frac{d}{d t} W_{n}\left(a_{\eta \xi}^{\prime}\right)(t)\right| \leq R_{\eta \xi, n}(t) \chi_{E}(t),
$$

for some subset $E$ of $[0, T]$ with measure $\mu(E) \leq \epsilon$.

Our claim (i) - (iv) above holds for the case $n=1$ from the definition of the maps $Y_{1}$ and $W_{1}$ and by applying Proposition (2.1). Assume that the claim holds for $n-1$, we show that it holds for $n$ as follows:

Choose $U_{n-1}(a)(t)(\eta, \xi) \in P\left(t, Y_{n-1}(a)(t)\right)(\eta, \xi)$ such that

$$
\begin{gathered}
\left|\frac{d}{d t} W_{n-1}\left(a_{\eta \xi}\right)(t)-U_{n-1}(a)(t)(\eta, \xi)\right| \\
=d\left[\frac{d}{d t} W_{n-1}\left(a_{\eta \xi}\right)(t), P\left(t, Y_{n-1}(a)(t)\right)(\eta, \xi)\right] \\
\leq D_{\eta \xi} K_{\eta \xi}^{P}(t) \frac{M_{\eta \xi}^{n-1}(t)}{(n-1) !}+\theta 2^{-n} K_{\eta \xi}^{P}(t) \sum_{i=0}^{n-1} \frac{\left(2 M_{\eta \xi}(t)\right)^{i}}{i !} .
\end{gathered}
$$

As $(\eta, \xi) \rightarrow U_{n-1}(a)(t)(\eta, \xi)$ is a sesquilinear form and $Y_{n-1}(a)$ is adapted, then there exists an adapted process $U_{n-1}(a):[0, T] \rightarrow \tilde{\mathcal{A}}$ such that

$$
U_{n-1}(a)(t)(\eta, \xi)=<\eta,\left(U_{n-1}(a)(t)\right) \xi>
$$

By inequality (3.9) and the assumption that $Y_{n-1}(a)$ lies in $L_{l o c}^{1}(\tilde{\mathcal{A}})$, then the process $U_{n-1}(a) \in$ $L_{l o c}^{1}(\tilde{\mathcal{A}})$ for each $a \in A$.

By (iv) of the recursive hypothesis, there exists $\delta_{n}>0$ such that $\left|a_{\eta \xi}^{\prime}-a_{\eta \xi}\right|<\delta_{n}$ implies

$$
\left|\frac{d}{d t} W_{n-1}\left(a_{\eta \xi}^{\prime}\right)(t)-\frac{d}{d t} W_{n-1}\left(a_{\eta \xi}\right)(t)\right| \leq R_{\eta \xi, n-1}(t) \chi_{E}(t)
$$

for some $E \subseteq[0, T]$ satisfying

$$
\int_{E} R_{\eta \xi, n-1}(t) d t \leq \theta 2^{-n-3}
$$

Next we define for any $a \in A, a_{\eta \xi} \in A(\eta, \xi)$

$$
\delta_{n}\left(a_{\eta \xi}\right)=\min \left\{\delta_{n}, \quad \theta 2^{-n-3}, \quad \frac{\left|a_{\eta \xi}-a_{\eta \xi, 0}\right|}{2}\right\}, a_{\eta \xi} \neq a_{\eta \xi, 0}
$$

and

$$
\delta_{n}\left(a_{\eta \xi, 0}\right)=\min \left\{\delta_{n}, \quad \theta 2^{-n-3}\right\} .
$$

We cover the set $A(\eta, \xi)$ with the balls $B\left(a_{\eta \xi}, \delta_{n}\left(a_{\eta \xi}\right)\right)$ and let $B\left(a_{\eta \xi, j}^{n}, \delta_{n}\left(a_{\eta \xi, j}^{n}\right)\right), j=0,1,2 \cdots m_{n}$, be a finite subcover, where we have put $a_{\eta \xi, 0}^{n}=a_{\eta \xi, 0}$. We remark that by the inequality

$$
\left|a_{\eta \xi, 0}-a_{\eta \xi, j}^{n}\right|>\frac{1}{2}\left|a_{\eta \xi, 0}-a_{\eta \xi, j}^{n}\right|
$$


the point $a_{\eta \xi, 0}$ belongs only to the ball $B\left(a_{\eta \xi, 0}, \delta_{n}\left(a_{\eta \xi, 0}\right)\right)$. Let $\left\{P_{j}^{n}(\cdot)\right\}_{j=0}^{j=m_{n}}$ be a continuous partition of unity subordinate to this covering and define the following intervals:

$$
I_{0}^{n}\left(a_{\eta \xi}\right)=\left[0, T P_{0}^{n}\left(a_{\eta \xi}\right)\right]
$$

and for $j>0$,

$$
I_{j}^{n}\left(a_{\eta \xi}\right)=\left[T\left(P_{0}^{n}\left(a_{\eta \xi}\right)+\cdots+P_{j-1}^{n}\left(a_{\eta \xi}\right)\right), \quad T\left(P_{0}\left(a_{\eta \xi}\right)+\cdots+P_{j}^{n}\left(a_{\eta \xi}\right)\right)\right] .
$$

Define the maps $Y_{n}, W_{n}$ as follows:

$$
\begin{gathered}
Y_{n}(a)(t)=a+\int_{0}^{t} \sum_{j=0}^{m_{n}} \chi_{I_{j}^{n}\left(a_{\eta \xi}\right)}(s) U_{n-1}\left(a_{j}^{n}\right)(s) d s \\
W_{n}\left(a_{\eta \xi}\right)(t)=a_{\eta \xi}+\int_{0}^{t} \sum_{j=0}^{m_{n}} \chi_{I_{j}^{n}\left(a_{\eta \xi}\right)}(s)<\eta, U_{n-1}\left(a_{j}^{n}\right)(s) \xi>d s
\end{gathered}
$$

Since $Y_{n-1}\left(a_{0}\right)=\Phi_{0}$ and by the properties of the process $U_{n-1}(a)$ and the fact that $I_{0}^{n}\left(a_{\eta \xi, o}\right)=$ $[0, T]$, we have

$$
Y_{n}\left(a_{0}\right)=\Phi_{0}
$$

and by Proposition $(2.1)$, the map $W_{n}: A(\eta, \xi) \rightarrow \operatorname{wac}(\tilde{\mathcal{A}})(\eta, \xi)$ given by $(3.12)$ is continuous and $W_{n}\left(a_{\eta \xi, 0}\right)=\Phi_{\eta \xi, 0}$. Furthermore, we have the following estimates:

$$
\begin{aligned}
& \int_{0}^{t}\left|\frac{d}{d s} W_{n}\left(a_{\eta \xi}\right)(s)-\frac{d}{d s} W_{n-1}\left(a_{\eta \xi}\right)(s)\right| d s \\
\leq & \int_{0}^{t} \sum_{j} \chi_{I_{j}^{n}\left(a_{\eta \xi}\right)}(s)\left|<\eta, U_{n-1}\left(a_{j}^{n}\right)(s) \xi>-\frac{d}{d s} W_{n-1}\left(a_{\eta \xi}\right)(s)\right| d s \\
\leq & \int_{0}^{t} \sum_{j} \chi_{I_{j}^{n}\left(a_{\eta \xi}\right)}(s)\left|<\eta, U_{n-1}\left(a_{j}^{n}\right)(s) \xi>-\frac{d}{d s} W_{n-1}\left(a_{\eta \xi, j}^{n}\right)(s)\right| d s \\
& +\int_{0}^{t} \sum_{j} \chi_{I_{j}^{n}\left(a_{\eta \xi}\right)}(s)\left|\frac{d}{d s} W_{n-1}\left(a_{\eta \xi, j}^{n}\right)(s)-\frac{d}{d s} W_{n-1}\left(a_{\eta \xi}\right)(s)\right| d s \\
\leq & \int_{0}^{t}\left(\sum_{j} \chi_{I_{j}^{n}\left(a_{\eta \xi}\right)}(s)\right)\left[D_{\eta \xi} K_{\eta \xi}^{P}(t) \frac{M_{\eta \xi}^{n-1}(t)}{n !}+\theta 2^{-n} K_{\eta \xi}^{P}(t) \sum_{i=0}^{n-1} \frac{\left(2 M_{\eta \xi}(t)\right)^{i}}{i !}\right] \\
& +\int_{0}^{t}\left(\sum_{j} \chi_{I_{j}^{n}\left(a_{\eta \xi}\right.}(s)\right) R_{\eta \xi, n-1}(s) \chi_{E}(s) d s \\
\leq & D_{\eta \xi} \frac{M_{\eta \xi}^{n}(t)}{n !}+\theta 2^{-n-1} \sum_{i=1}^{n} \frac{\left(2 M_{\eta \xi}(t)^{i}\right.}{i !}+\theta 2^{-n-3} .
\end{aligned}
$$

Hence, item (i) of the recursive hypothesis holds for all n. Next we fix $t \in[0, T]$ and let $j$ be such that $t \in I_{j}\left(a_{\eta \xi}\right)$. Then by (3.11) and (3.12),

$$
\begin{aligned}
& d\left(\frac{d}{d t} W_{n}\left(a_{\eta \xi}\right)(t), P\left(t, Y_{n-1}(a)(t)\right)(\eta, \xi)\right) \\
= & d\left(<\eta, U_{n-1}\left(a_{j}^{n}\right)(t) \xi>, P\left(t, Y_{n-1}(a)(t)\right)(\eta, \xi)\right) \\
\leq & \rho\left(P \left(t, Y_{n-1}\left(a_{j}^{n}\right)(t)(\eta, \xi), P\left(t, Y_{n-1}(a)(t)(\eta, \xi)\right)\right.\right. \\
\leq & K_{\eta \xi}^{P}(t)\left\|Y_{n-1}\left(a_{j}^{n}\right)(t)-Y_{n-1}(a)(t)\right\|_{\eta \xi} .
\end{aligned}
$$


By applying (3.10), we have

$$
\begin{aligned}
& \left\|Y_{n-1}\left(a_{j}^{n}\right)(t)-Y_{n-1}(a)(t)\right\|_{\eta \xi} \\
& \leq\left|a_{\eta \xi, j}^{n}-a_{\eta \xi}+\int_{0}^{t}\right| \frac{d}{d s} W_{n-1}\left(a_{\eta \xi, j}^{n}\right)(s)-\frac{d}{d s} W_{n-1}\left(a_{\eta \xi}\right)(s) \mid d s \\
& \leq \theta 2^{-n-3}+\theta 2^{-n-3}=\theta 2^{-n-2}
\end{aligned}
$$

Combining inequalities (3.13) and (3.14), we have

$$
d\left(\frac{d}{d t} W_{n}\left(a_{\eta \xi}\right)(t), P\left(t, Y_{n-1}(a)(t)\right)(\eta, \xi)\right) \leq \theta 2^{-n-2} K_{\eta \xi}^{P}(t) .
$$

The estimate is independent of $j$ and so it holds on the whole interval $[0, T]$. This proves item (ii) of our claim. To establish item (iii) we proceed as follows:

$$
\begin{aligned}
& d\left(\frac{d}{d t} W_{n}\left(a_{\eta \xi}\right)(t), P\left(t, Y_{n}(a)(t)\right)(\eta, \xi)\right) \\
& \leq d\left(\frac{d}{d t} W_{n}\left(a_{\eta \xi}\right)(t), P\left(t, Y_{n-1}(a)(t)\right)(\eta, \xi)\right) \\
& +\rho\left(P\left(t, Y_{n-1}(a)(t)\right)(\eta, \xi), P\left(t, Y_{n}(a)(t)\right)(\eta, \xi)\right)
\end{aligned}
$$$$
\leq \theta 2^{-n-2} K_{\eta \xi}^{P}(t)+K_{\eta \xi}^{P}(t)\left\|Y_{n-1}(a)(t)-Y_{n}(a)(t)\right\|_{\eta \xi}
$$$$
\leq \theta 2^{-n-2} K_{\eta \xi}^{P}(t)+K_{\eta \xi}^{P}(t) \int_{0}^{t}\left|\frac{d}{d s} W_{n-1}\left(a_{\eta \xi}\right)(s)-\frac{d}{d s} W_{n}\left(a_{\eta \xi}\right)(s)\right| d s
$$$$
\leq K_{\eta \xi}^{P}(t)\left[\theta 2^{-n-2}+D_{\eta \xi} \frac{M_{\eta \xi}^{n}(t)}{n !}+\theta 2^{-n-1} \sum_{i=1}^{n} \frac{\left(2 M_{\eta \xi}(t)\right)^{i}}{i !}+\theta 2^{-n-3}\right] \text {, by }(i) \text { and }(i i)
$$$$
\leq D_{\eta \xi} K_{\eta \xi}^{P}(t) \frac{M_{\eta \xi}^{n}(t)}{n !}+\theta 2^{-n-1} K_{\eta \xi}^{P}(t) \sum_{i=0}^{n} \frac{\left(2 M_{\eta \xi}(t)\right)^{i}}{i !}
$$

(obtained by applying the inequality $; \theta 2^{-n-2}+\theta 2^{-n-3} \leq \theta 2^{-n-1}$ ).

The last estimate proves item (iii) of our claim. Item (iv) of the claim is established by the application of Proposition $(2.1)$ to the map $W_{n}: A(\eta, \xi) \rightarrow \operatorname{wac}(\tilde{\mathcal{A}})(\eta, \xi)$.

From item (i), we have

$$
\begin{aligned}
& \left|Y_{n}(a)-Y_{n-1}(a)\right|_{\eta \xi} \\
& =\left\|Y_{n}(a)(0)-Y_{n-1}(a)(0)\right\|_{\eta \xi}+\int_{0}^{T}\left|\frac{d}{d t}<\eta, Y_{n}(a)(t) \xi>-\frac{d}{d t}<\eta, Y_{n-1}(a)(t) \xi>\right| d t \\
& =\left|W_{n}\left(a_{\eta \xi}\right)(0)-W_{n-1}\left(a_{\eta \xi}\right)(0)\right|+\int_{0}^{T}\left|\frac{d}{d s} W_{n}\left(a_{\eta \xi}\right)(s)-\frac{d}{d s} W_{n-1}\left(a_{\eta \xi}\right)(s)\right| d s \\
& \leq D_{\eta \xi} \frac{M_{\eta \xi}^{n}(T)}{n !}+\theta 2^{-n-1} e^{2 M_{\eta \xi}(T)}
\end{aligned}
$$


It follows by (3.15) that the sequence $\left\{Y_{n}(a)\right\}$ is uniformly Cauchy in $\operatorname{wac}(\tilde{\mathcal{A}})$ and thus converges uniformly to a map $\Phi: A \rightarrow \operatorname{wac}(\tilde{\mathcal{A}})$. Again,

$$
\lim _{n \rightarrow \infty} W_{n}\left(a_{\eta \xi}\right)(t)=\lim _{n \rightarrow \infty}<\eta, Y_{n}(a)(t) \xi>=<\eta, \Phi(a)(t) \xi>
$$

The map $a_{\eta \xi} \rightarrow<\eta, \Phi(a)(t) \xi>$ is continuous since the map $a_{\eta \xi} \rightarrow W_{n}\left(a_{\eta \xi}\right)$ is continuous for each $n$.

As the stochastic process $\Phi(a)$ is the limit of a sequence of adapted weakly absolutely continuous processes in $L_{\text {loc }}^{1}(\tilde{\mathcal{A}}), \Phi(a)$ lies in $\operatorname{Ad}(\tilde{\mathcal{A}})_{w a c} \cap L_{l o c}^{1}(\tilde{\mathcal{A}})$ and $\Phi\left(a_{0}\right)=\Phi_{0}$.

By (iii) of the recursive formula,

$$
d\left(\frac{d}{d t}<\eta, \Phi(a)(t) \xi>, P(t, \Phi(a)(t))(\eta, \xi)\right)=0 .
$$

Therefore

$$
\Phi(a) \in \mathrm{S}^{(T)}(a), \quad<\eta, \Phi(a)(\cdot) \xi>\in \mathrm{S}^{(T)}(a)(\eta, \xi), \quad<\eta, \Phi\left(a_{0}\right)(\cdot) \xi>=\Phi_{\eta \xi, o}
$$

The following corollaries show that the set map $S^{(T)}(a)(\eta, \xi)$ and the set of complex numbers $R^{(T)}(a)(\eta, \xi)$ associated with the reachable set of QSDI (1.1) at the final time $T$ admit some continuous parametrizations.

\section{Corollary 3.2.}

There exists a subspace $\mathcal{U}$ of the space of all continuous maps from $A(\eta, \xi)$ into $\operatorname{wac}(\tilde{\mathcal{A}})(\eta, \xi)$ and a continuous function

$$
g: A(\eta, \xi) \times \mathcal{U} \rightarrow \operatorname{wac}(\tilde{\mathcal{A}})(\eta, \xi)
$$

such that for any $a \in A, \quad a_{\eta \xi} \in A(\eta, \xi)$,

$$
g\left(a_{\eta \xi}, \mathcal{U}\right)=S^{(T)}(a)(\eta, \xi)
$$

Proof. We remark first that the $\operatorname{space} \operatorname{wac}(\tilde{\mathcal{A}})(\eta, \xi)$ is a subspace of the space $A C[0, T]$ of all absolutely continuous complex valued functions on $[0, T]$, a separable Banach space with the usual sup norm.

We put $X$ to be the set of continuous maps from the compact set $A(\eta, \xi)$ into $\operatorname{wac}(\tilde{\mathcal{A}})(\eta, \xi)$ and define the subspace $\mathcal{U}$ of $X$ by

$$
\mathcal{U}=\left\{W: A(\eta, \xi) \rightarrow \operatorname{wac}(\tilde{\mathcal{A}})(\eta, \xi) / W \text { is continuous and } W\left(a_{\eta \xi}\right) \in S^{(T)}(a)(\eta, \xi)\right\}
$$

the set of all continuous selections from the map $a_{\eta \xi} \rightarrow S^{(T)}(a)(\eta, \xi)$.

Define the map $g$ by

$$
g\left(a_{\eta \xi}, W\right)=W\left(a_{\eta \xi}\right)
$$


Then by the continuity of each $W \in \mathcal{U}, g$ is continuous and by Theorem 3.1 above,

$$
g\left(a_{\eta \xi}, \mathcal{U}\right)=S^{(T)}(a)(\eta, \xi)
$$

\section{Corollary 3.3.}

There exists a subset $\mathcal{U}$ of the space of all continuous maps from $A(\eta, \xi)$ into $\operatorname{wac}(\tilde{\mathcal{A}})(\eta, \xi)$ and a continuous function

$$
h: A(\eta, \xi) \times \mathcal{U} \rightarrow \mathbb{C}
$$

such that for any $a \in A, a_{\eta \xi} \in A(\eta, \xi)$

$$
h\left(a_{\eta \xi}, \mathcal{U}\right)=R^{(T)}(a)(\eta, \xi)
$$

Proof . Adopting the notation employed in the proof of Corollary 3.2, we define the map $h\left(a_{\eta \xi}, W\right)=W\left(a_{\eta \xi}\right)(T)$.

Since $W\left(a_{\eta \xi}\right) \in S^{(T)}(a)(\eta, \xi), W\left(a_{\eta \xi}\right)$ is of the form $W\left(a_{\eta \xi}\right)=<\eta, \tilde{W}(\cdot) \xi>$ for some $\tilde{W} \in$ $S^{(T)}(a)$, we have

$$
W\left(a_{\eta \xi}\right)(T)=<\eta, \tilde{W}(T) \xi>\in R^{(T)}(a)(\eta, \xi)
$$

By Theorem 3.1, we have

$$
h\left(a_{\eta \xi}, \mathcal{U}\right)=R^{(T)}(a)(\eta, \xi)
$$

\section{ACKNOWLEDGEMENTS}

I am grateful to the Abdus Salam International Centre for Theoretical Physics, Trieste, Italy for my appointment as a Regular Associate of the Centre and to the Swedish International Development Agency (SIDA) for providing funds to support the appointment. The results presented in this paper were obtained during one of my visits to the Centre under the Associateship programme.

\section{REFERENCES}

Andrezej Fryszkowski (1983). Continuous selections for a class of non- convex multivalued maps; Studia Mathematica, T. LXXVI, 163 - 174.

Antosiewicz, H. A; Cellina, A. (1975). Continuous selections and differential relations, Journal of Differential Equations 19, 386 - 398.

Arrigo Cellina (1988) . On the set of solutions to Lipschitzian differential inclusions . Differential and Integral Equations, 1(4), 495 - 500.

Arrigo Cellina; Antonio Ornelas (1992). Representation of the attainable set for Lipschitzian differential inclusions Rocky Mountain Journal of Mathematics, 22(1), 117 - 124.

Aubin, J. P; Cellina, A.(1984) Differential Inclusions, Springer - Verlag, Berlin.

Ayoola, E. O. (2003a). Error estimates for discretized quantum stochastic differential inclusions, Stochastic Analysis and Applications, 21(6) 1215 - 1230. 
Ayoola, E.O. (2003b). Exponential formula for the reachable sets of quantum stochastic differential inclusions, Stochastic Analysis and Applications 21(3), 515 - 543.

Ayoola, E. O. (2001). Construction of approximate attainability sets for Lipschitzian quantum stochastic differential inclusions, Stochastic Analysis and Applications, 19(3), $461-471$.

Colombo, R. M, Fryszkowski, A; Rzezuchowski, T; Staicu, V. (1991) Continuous selections of solution sets of Lipschitzian differential inclusions, Funkcialaj Ekvacioj 34321 - 330.

Ekhaguere, G. O. S.(1992). Lipschitzian quantum stochastic differential inclusions, International Journal of Theoretical Physics Vol.31(11), 2003-2034.

Ekhaguere, G. O. S.(1995). Quantum stochastic differential inclusions of hypermaximal monotone type, International Journal of Theoretical Physics, 34(3), 323-353.

Ekhaguere, G.O.S.(1996). Quantum stochastic evolutions, International Journal of Theoretical Physics 35(9) 1909-1946.

Georgi V. Smirnov (2002). Introduction to the Theory of Differential Inclusions, Graduate Studies in Mathematics, volume 41 American Mathematical Society, Providence, RI.

Halmos, P. R. (1988). Measure Theory. Graduate Texts in Mathematics, Springer - Verlag.

Hudson, R. L; Parthasarathy, K. R. (1984). Quantum Ito's formulae and stochastic evolutions, Communications in Mathematical Physics, 93, 301-324

Mireille Broucke; Ari Arapostathis (2001). Continuous interpolation of solutions of Lipschitz inclusions Journal of Mathematical Analysis and Applications, 258, 565 - 572.

Repovs, D; Semenov, P.V. (1998). Continuous Selections of Multivalued Mappings, Mathematics and its Applications, Kluwer, Dordrecht. 